\title{
Microwave Edge Modes on a Metasurface With Glide Symmetry
}

\author{
Julia D de Pineda, ${ }^{*}$ Alastair P Hibbins, and J Roy Sambles \\ Department of Physics and Astronomy, University of Exeter, Exeter EX4 4QL, United Kingdom.
}

(Dated: March 6, 2019)

\begin{abstract}
In this work we study planar metasurfaces comprised of two layers of hexagonal arrays of circular metal patches. This two-layer geometry supports a bound surface wave that propagates along the $\mathrm{x}$-direction. In our study, each metasurface is infinitely periodic in one direction $(\mathrm{x})$ but only a few periods wide in the orthogonal direction $(\mathrm{y})$. Through experiments and modelling we find evidence of a localized edge mode whose existence is dependent on the relative alignment of the two layers. This edge mode is used to guide the propagation of electromagnetic energy around both triangular and hexagonal shapes.
\end{abstract}

\section{INTRODUCTION}

Conductors support the propagation of electromagnetic waves localized at their interface with an insulator. For visible frequencies these modes are known as surface plasmons and have been widely studied since the 1950s [1]. Perfect conductors (e.g. metals at microwave frequencies) are unable to support bound surface modes since the fields are completely excluded from the substrate, however patterning of surfaces on a scale-length similar or smaller than the excitation wavelength recovers a similar 'plasmonic' phenomena. This concept was familiar to radar engineers of the mid-twentieth century $[2,3]$, and was readdressed theoretically and experimentally [4, 5] six decades later. In 2004 Pendry [4] showed that the dispersion of the modes supported on perfect conductors perforated with an array of subwavelength holes is governed by an effective permittivity of the same form as the plasmonic analogy. This behaviour is not only limited to holey structures but can also be extended to several more surface textures that provide different interesting properties to the structures. The size and spacing of the texturing elements can readily be controlled over a wide range of length scales, which allows the creation of designer surface waves with almost arbitrary dispersion in frequency and in space. These textured structures that support the propagation of surface modes are known as metasurfaces.

Metasurfaces and their use to manipulate electromagnetic fields have significant end-user interest due to their low profile and low cost. Their applications to guide surface waves [6] and to transform them into different wave field configurations with desirable properties [7] is of particular commercial potential. The electromagnetic properties (i.e. surface impedance, mode shape) of metasurfaces can easily be controlled across the structure by simply varying the size or the shape of its elements [7], making them ideal for the manufacture of graded index devices such as lenses and leaky wave antennas [8-10].

Over the last few years, the study of metasurfaces with high symmetries, first proposed by Hessel et al. in 1973

\footnotetext{
* jd602@exeter.ac.uk
}

[11], has gained popularity. Examples of high symmetries applied to electromagnetic metasurface problems include 'twist' symmetry [12] and 'glide' symmetry [13]. In glide symmetry, one element is translated by half its period and then mirrored in a glide plane. Similarly, in a 'p-fold' twist-symmetry, the elements are translated and rotated around an axis. Both symmetries reduce the dispersion of modes supported by metasurfaces, contributing to solve one of the major problems presented by metasurfaces due to the the resonant nature and the periodicity of the structure. For structures presenting the mentioned symmetries, the expected band gap between the first and second modes at the first Brillouin zone boundary is closed and the dispersion diagram of the first mode is almost linear, resulting in an increased bandwidth [14-18].

In this paper, we present a finite sized metasurface with glide symmetry. It consists of a bi-layer metallic structure comprised of two simple metasurfaces. The metasurfaces are identical to one another, and effectively two-dimensional as their thickness is three orders of magnitude smaller than the lateral dimensions of the sample. Because of their hexagonal lattice, the structure has the peculiarity of supporting the edge mode in only one of the two possible high symmetry arrangements of the two layers, which we explore in detail through experimentally determined band structure and modelled field distributions. We also demonstrate how this edge mode can be used to guide the electromagnetic fields in different configurations. Guiding energy on surfaces, such as steering them around sharp bends and splitting and redirecting energy between channels is of relevance to antenna and communication engineers, and also has fundamental interest to the physics community [19-21]. For those applications, metasurfaces that support the propagation of edge modes have been studied in the past years [22-25]. In contrast to the case presented in [22], where the edge mode appears as the amount of dielectric present in the metasurface is changed as the edge is modified, in this work, we keep the ratio of dielectric/metal invariant but displace one layer with respect to the other. This simply means that the same physical structure can be chosen to either support the propagation of the edge mode or not by simply shifting one of the layers that comprise it. 


\section{DESCRIPTION AND ANALYSIS OF THE METASURFACE}

The metasurface under study here is comprised of two layers of circular copper patches in a hexagonal array separated by a small dielectric spacing. Both layers are identical but have been displaced with respect to each other in the plane of the structure. The patches are printed over a dielectric slab that also acts as a spacing between both layers. In Fig. 1 a unit cell of the infinite structure is presented. The top layer (red) has been displaced by $\frac{p}{\sqrt{3}}$ in the $\mathrm{x}$-direction with respect to the bottom layer (grey), where $p=2.4 \mathrm{~mm}$ is the lattice spacing of the structure. The circular metallic patches are made of copper, have a diameter $d=2.15 \mathrm{~mm}$ and are printed over a dielectric slab of permittivity $\varepsilon=2.8$ with a thickness of $t=25 \mu \mathrm{m}$.

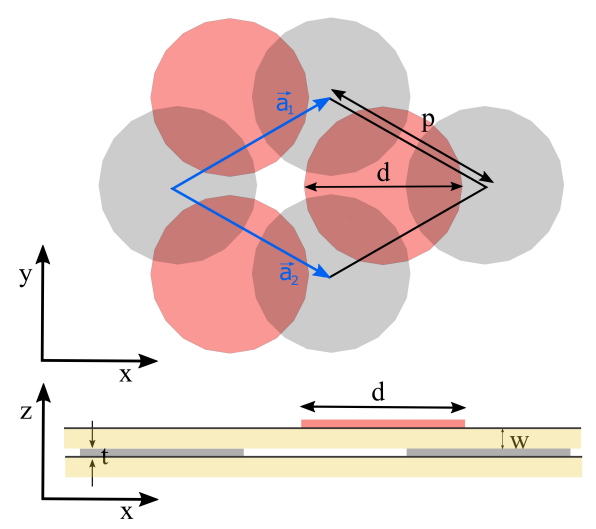

FIG. 1. a) Unit cell (rhombic shape) of the structure comprised of copper patches printed over a dielectric slab. Each layer is represented by a different color (red and grey) and the dielectric is represented in yellow. The diameter of the patches is $d=2.15 \mathrm{~mm}$ and the periodicity of the structure is $p=2.4 \mathrm{~mm}$. The dielectric thickness is $w=25 \mu \mathrm{m}$ and the copper thickness is $t=18 \mu \mathrm{m}$. The blue arrows represent the unit vectors of the structure in real space.

This metasurface is electrically disconnected, and therefore supports bound surface modes whose fields are predominantly transverse electric (TE) in nature at low frequencies [26]. The lowest energy mode is a hybridization of a grazing plane wave with the resonance supported in the dielectric between the overlapping (upper and lower) regions of metal. In this space, the electric field is orientated in the z-direction and confined to the overlap regions while outside the bi-layer, the field is in the plane of the surface, but transverse with respect to the propagation direction. The magnetic field has a component parallel to the direction of propagation and describes loops between adjacent patches. Strong confinement of the energy associated with the fields in the high permittivity dielectric in the overlap regions leads to an increase of the mode index (reduction of phase velocity) with respect to the single layer structure. The Eigenmodes supported by the infinitely periodic bi-layer metasurface have been calculated using Finite Element Method (FEM) modelling. Floquet boundary conditions are imposed in the model to simulate the solutions as a function of wave-vector, yielding the full in-plane surface-wave dispersion (Fig. 2). The dispersion has also been measured experimentally. Samples are manufactured using the lithographic technique described in [9]. The layers were etched separately, and then aligned, using a thin layer of oil placed in between, to exploit the surface tension to laminate them together. The oil is included in the model as a layer with thickness $t=25 \mu \mathrm{m}$ and a permittivity of $\varepsilon=3.4$.

Experimentally, the modes are excited and detected with identical magnetic-field $(\mathrm{H})$ antennas. They are formed from RF coaxial cables whose insulating sheath are partially removed. A small loop (radius, $0.5 \mathrm{~mm}$ ) antenna is then soldered to the exposed signal line and to the earth shielding. These loop antennas are each fed to ports on a $40 \mathrm{GHz}$ Anritsu vector network analyzer (VNA). The antennas are placed $0.5 \mathrm{~mm}$ away from the sample. With the source kept fixed in position, the probe is used to scan an area on the opposite face of the sample in steps of $1.0 \mathrm{~mm}$, allowing enough time between measurements for the probe to stop vibrating after it stops moving.

The feeding and detection antennas have not been optimized in terms of insertion loss since device-design is not of immediate concern for this study, instead we wish to understand the band-structure of the supported modes. To achieve this, the VNA measures the amplitude and phase of the electromagnetic fields (referenced internally to the source), and we apply a 2D Fourier Transform to the recorded data. Fig. 2 illustrates these results compared with the predictions from the FEM model.

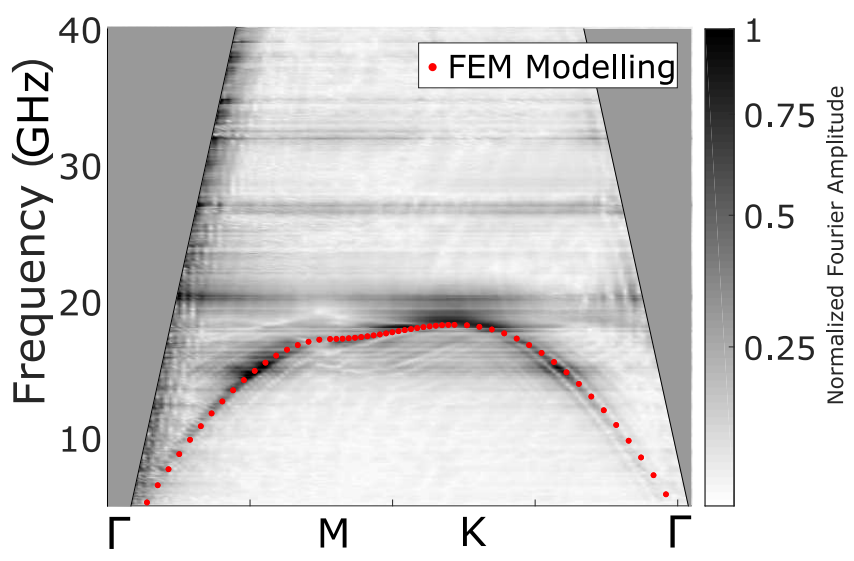

FIG. 2. Dispersion of the modes supported by an infinitely periodic tessellation of the unit cell shown in Fig. 1. The grey-scale represents the amplitude of the Fourier coefficients of the Fourier-transformed field-data. The red dots are the Eigenmodes predicted by the FEM modelling, using a patch diameter $d=2.15 \mathrm{~mm}$ and a periodicity of the structure $p=$ $2.4 \mathrm{~mm}$. The dielectric thickness is $w=25 \mu \mathrm{m}$ and the copper thickness is $t=18 \mu \mathrm{m}$. 
While a single layer of patches also supports surface modes [7], the modes supported in the bi-layer array are much more localized, confined to the high-index medium, and therefore disperse more strongly from the light-line even at the lowest frequencies [9].

\section{1D PERIODIC STRIPS}

In this work we explore finite-width strips of the bilayer metasurface previously described. The experimental samples are $250 \mathrm{~mm}$ long in the propagation direction, and are modelled as infinitely periodic using Floquet boundary conditions in the FEM model. Since the structure is of finite width, it is only periodic in the propagation direction: it therefore presents a rectangular unit cell. Our chosen bi-layer metasurface has patches in an hexagonal lattice in each layer, and is 4 patches in width. It has two possible arrangements, yielding two different edge-geometries, as shown in Fig. 3. Specifically, one arrangement (a) has two overlap regions per edge patch, while the other (b), has just one overlap region per edge patch. In both cases, the bulk metasurface remains the same. The different arrangements correspond to mode propagation directions $30^{\circ}$ apart in the infinitely periodic lattice.
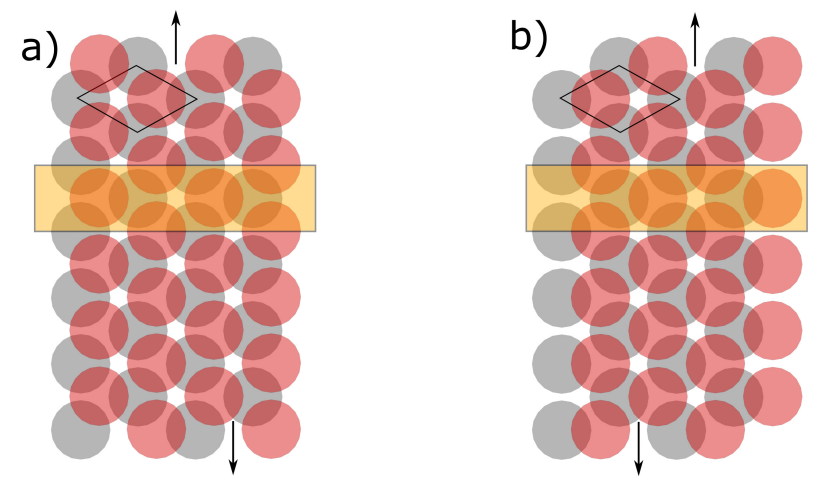

FIG. 3. Representation of the 4-patch-wide strips of the 2layer hexagonal structure. The top layer is represented in red and the bottom layer is represented in grey. A rhombic unit cell of the infinite structure is marked in black. The area highlighted in yellow corresponds to the unit cell of the finite strip. a) shows the structure terminated with two overlap region per edge patch while b) shows the structure terminated with one overlap regions per edge patch. Both structures have a patch diameter of $d=2.15 \mathrm{~mm}$ and the periodicity of the structure is $p=2.4 \mathrm{~mm}$

The Eigenmodes of both structures have been obtained using FEM software, and we plot their band structure (dispersion) and field distributions in Fig. 4. Since the bulk metasurface is identical for both structures, we associate any differences in the Eigenmodes supported with the edge-geometries. While for the structure with two overlap regions per edge patch, (a), there is a family of three modes (red lines) that become degenerate at the Brillouin Zone boundary (identified as bulk 'surface' modes according to the field plots), the geometry with just one overlap region per edge patch, (b), also supports two higher energy modes (blue lines).

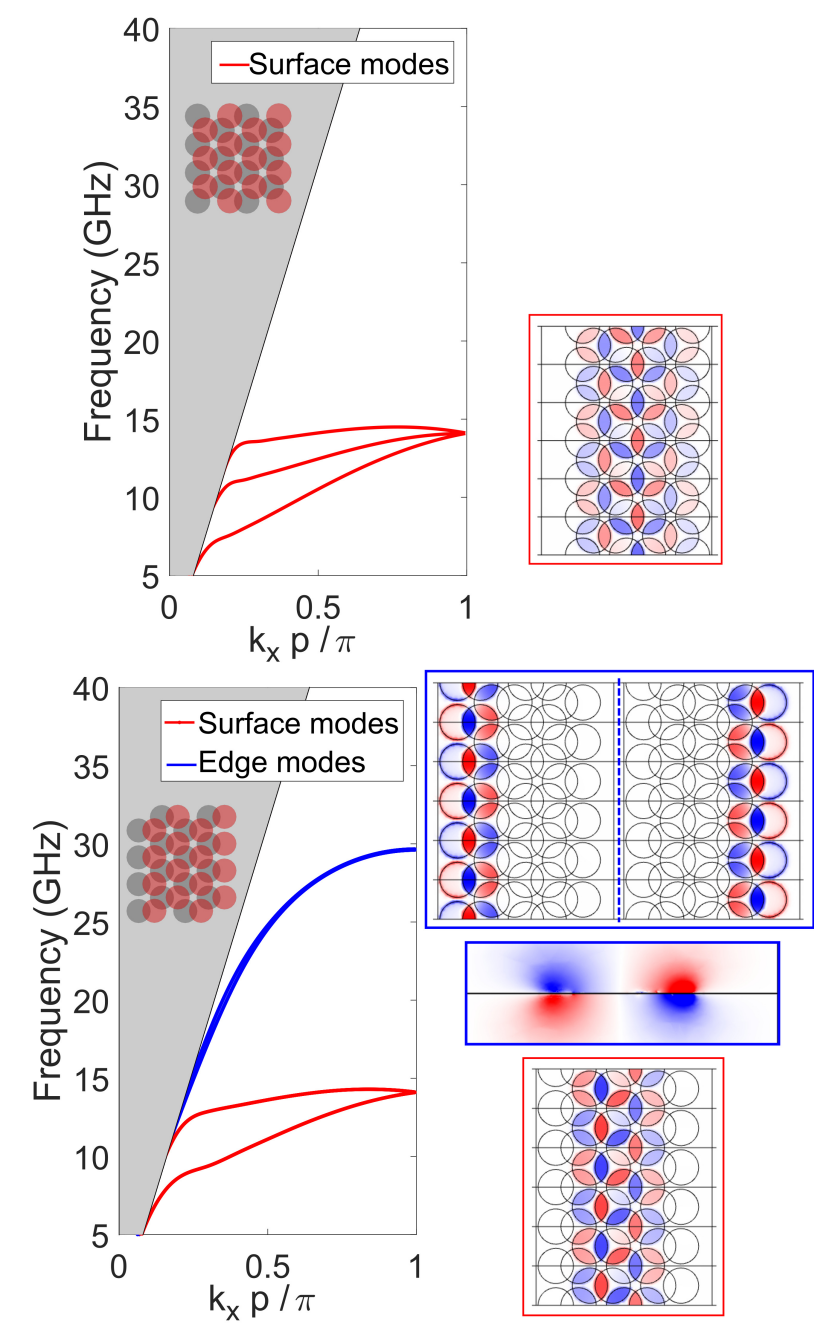

FIG. 4. Dispersion diagrams and field plots for the structures presented in Fig. 3 The top plots correspond to Fig. 3 (a): the structure with two overlap regions per edge patch. The bottom plots correspond to Fig. 3 (b): the structure with one overlap region per edge patch. In the dispersion diagrams the surface modes are represented in red while the edge modes are represented in blue and the radiative zone is shaded in grey. In the field plots the outline of the patches that comprise the structure are represented with black lines. Note that the two edge modes in (b) are degenerate. For this second case, a vertical cross-section of the fields corresponding to the edge modes has also been included.

These two extra degenerate modes in Fig. 4 (b) are identified as edge modes according to the field plots as they are only allowed to propagate along the interface between the structure and the surrounding dielectric. A splitting in frequency is observed for the bulk modes, this is caused by the quantization in the transverse direction due to the finite size of the sample [27]. The number of 
bulk modes depends on the number of bulk 'meta-atoms' present in the structure and it does not correspond to the number of patches in each row since the behaviour of the structure is not determined by the resonances of the patches, but the overlap between them. Hence the surface's meta-atoms are formed by two patches, one in the top layer and one in the bottom layer and each patch must have three overlap regions. For the unit cell of the first structure (Fig. 3 (a)) there are three of these metaatoms and hence three surface modes are found, whereas, for the structure in Fig. 3 (b) due to the presence of the edge patches with a different environment, only two surface modes are found. As for the existence of supported edge modes, an essential condition for the appearance of an edge mode is the presence of isolated charges on the edge of the structure. Another condition that needs to be fulfilled is that the entire structure is neutral. Therefore, because the structure is comprised of disconnected patches, every individual patch needs to remain neutral. For the structure in Fig. 3 (a) this condition is fulfilled with no need for the edges of the patches to be charged as the two different overlap regions in the patch can have opposite sign charges. In contrast, for the structure in Fig. 3 (b), there is only one overlap region per edge patch which is charged either positively or negatively. In that way, in order for the patch to remain neutral there must be a distribution of charges along the outside edge of the structure. The mentioned distribution can be seen in the field plots represented in Fig. 4.
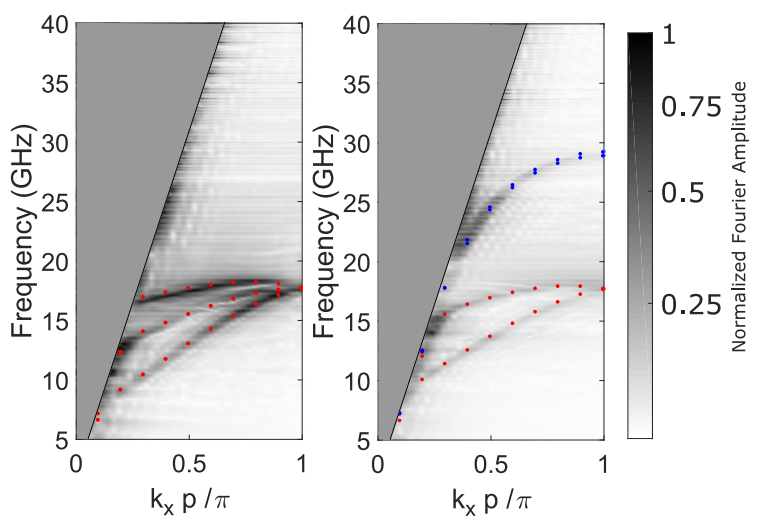

FIG. 5. Experimental dispersion diagrams for the structures presented in Fig.3 The plot on the left corresponds to the structure with two overlap regions at the edge patches, so it does not present an edge mode and the plot on the right is for the structure with one overlap region at the edge patches. The modelled dispersion is also represented (red dots for the surface modes and blue dots for the edge modes), showing excellent agreement with the experimental measurement.

An experimental confirmation of the dispersion diagrams presented in Fig. 4 has also been realized. Two samples have been fabricated and measured using the same technique as described before for the infinite sample. The metal patches that form the strips are printed over a large $(30 \mathrm{~cm}$ by $40 \mathrm{~cm})$ sheet of dielectric, resulting in a sample that is much wider than the studied strips $(1.5 \mathrm{~cm})$. In this way, the edges of the sample, where the oil layer can present a curved edge are far away from the region of interest.

The dispersion diagrams obtained experimentally match those obtained with the finite element modelling software, as shown in Fig. 5. Again, a layer of oil has been added to the FEM model to resemble the experimental sample. While the surface modes are strongly dependent on the thickness of the sample, moving up in frequency as the separation of the two layers is increased, the edge modes show only weak dependence. This is because the surface modes are determined by the capacitive effect between the patches in both layers whereas the edge modes are determined by the charges accumulated at the edges of the terminating patches. This can be observed by comparing the plots in Fig. 4 (no oil layer added to the model) and Fig. 5, where the separation between the layers is increased by adding the oil layer. While the surface modes move up in frequency when the oil layer is introduced, the edge modes stay in the same position. From the experimental measurements, some properties relative to the propagation of the edge mode such as the mode index and the decay length can be obtained. In the range of frequencies where the edge mode is supported, the effective mode index varies between 1.11 at $19 \mathrm{GHz}$ and 1.30 at $25 \mathrm{GHz}$ while the decay constant of the mode, normalized to the free space wave vector varies between 0.014 and 0.023 over the same range of frequencies. This means that the wave has to travel for more than 50 wavelengths before its amplitude is reduced by a factor of e. The measured mode index and normalized decay constant for the studied range of frequencies are shown in Appendix A.

\section{USING THE EDGE MODE TO GUIDE THE ELECTROMAGNETIC FIELD}

A second experiment was subsequently carried out in order to confirm the edge nature of the higher frequency modes found for the structure in Fig. 4 (b) and to test the capability of the edge mode to propagate around corners. Two different samples were studied, one with a hexagonal shape $\left(240^{\circ}\right.$ corners $)$ and a triangular one $\left(300^{\circ}\right.$ corners), etched from a meta-coated dielectric sheet. These samples are designed in a way that all the edges present the necessary geometry that allows the edge Eigenmode, with one overlap region per edge patch. While for the triangular shape all three edges have the same orientation, for the hexagonal one the edges alternate between 'up' edges and 'down' edges. To excite the edge mode, a loop antenna is placed in one corner of the structures and a second loop antenna was used to measure the amplitude and phase of the electromagnetic fields across the entire opposite face of the sample. The measured fields for four different frequencies are plotted in Fig. 6.

For low frequencies (i.e. $12 \mathrm{GHz}$ ), where there exists 

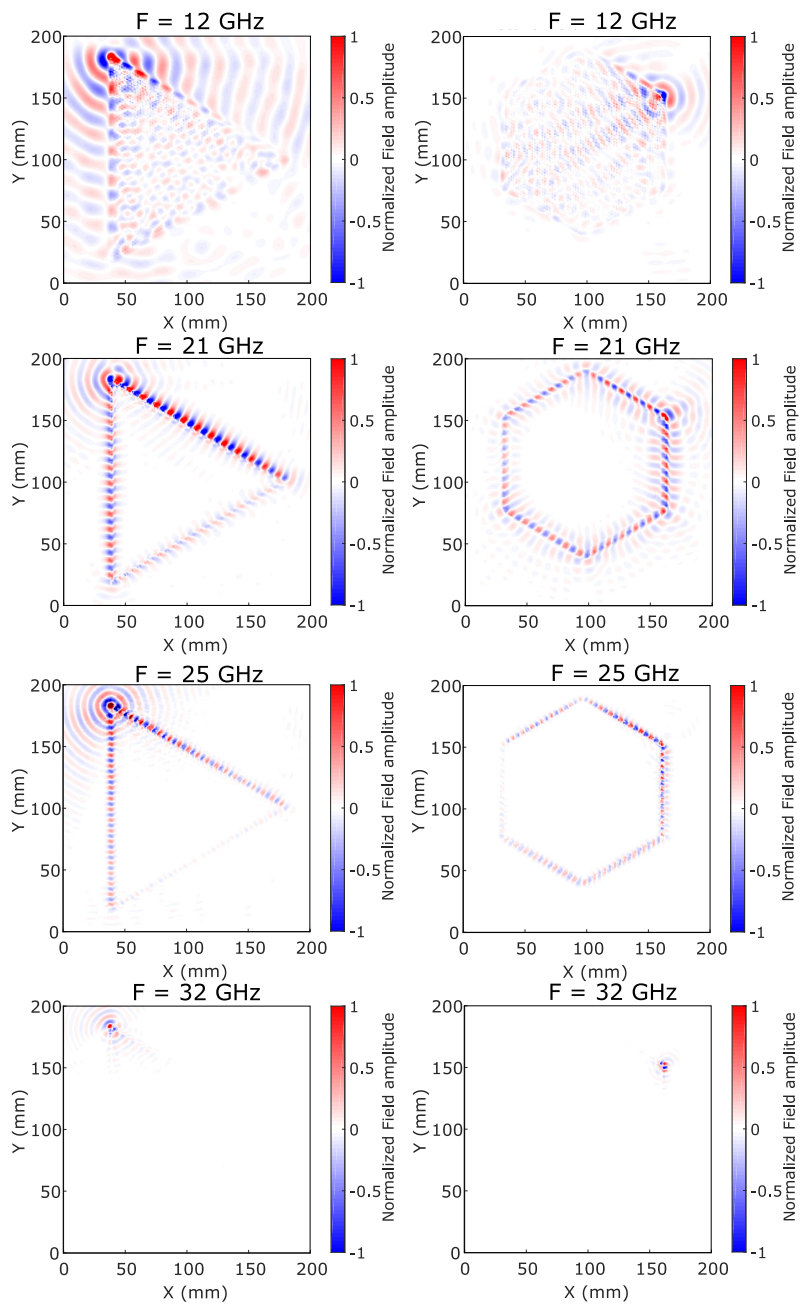

FIG. 6. Experimental measurement of the fields on top of the sample with the triangular shape (left) and the hexagonal shape (right). The triangular structure is excited on the top left corner and the hexagonal one on the top right corner. For low frequencies (i.e. $12 \mathrm{GHz}$ ) the field penetrates inside the structure. For higher frequencies (i.e. $21 \mathrm{GHz}$ and $25 \mathrm{GHz}$ ), where only the edge mode exists, the field propagates around the edges and for frequencies above the cutoff of the modes supported by the structure (i.e. $32 \mathrm{GHz}$ ) we do not find any modes bound to the surface.

a surface mode, the fields penetrate inside the structure and the mode propagates across the entire metasurface. For higher frequencies, (i.e. $21 \mathrm{GHz}$ and $25 \mathrm{GHz}$ ) where only the edge mode exists, the mode propagates around the triangular/hexagonal shape with a rapid evanescent decay of the fields across the surface. Finally, for frequencies above the cutoff of the edge mode (i.e. $32 \mathrm{GHz}$ ) we cannot find any mode bound to the edge or surface. This experiment demonstrates the edge nature of the additional mode supported by the described structure and its capability to guide the electromagnetic fields, even around sharp corners.

Finally an inside-edge sample, complementary to the previously studied hexagonal shape was fabricated and

studied. In this case, a hexagonal hole has been cut out from an infinite 2 layer structure, leaving around the shape an edge-mode-supporting configuration of patches. The edge mode was excited by placing a loop antenna on the top corner of the hexagonal shape and, using the same experimental setup, the electromagnetic field was measured across the opposite surface of the sample. In Fig. 7 a comparison of the field plots for both hexagonal structures is shown for two different frequencies inside the range where the edge mode is supported. It is proven that the edge mode is able to propagate around both configurations even in the presence of sharp corners. For the hexagonal shape, the mode propagates around three corners and for a distance of more than 20 wavelengths. The signal radiated as the wave goes around a corner has also been quantified and is smaller than $30 \%$ for frequencies between $19 \mathrm{GHz}$ and $22 \mathrm{GHz}$. The percentage of the amplitude that is lost at the corner for different frequencies is shown in Fig. 8 in Appendix A.

a)
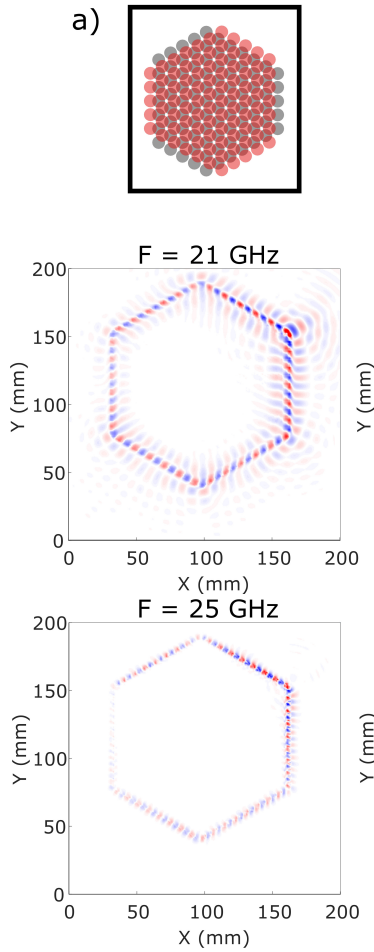

FIG. 7. Experimental measurement of the fields on top of the sample with the hexagonal shape (left) and the hexagonal hole (right). Both structures have been excited at the top right corner with a loop antenna. The field plots are shown at $21 \mathrm{GHz}$ and $25 \mathrm{GHz}$. For both frequencies, only the propagation of the edge mode is supported.

\section{CONCLUSION}

A double layered hexagonal metal-patch structure with glide symmetry has been modelled, fabricated and exper- 
imentally characterized. Whereas the infinitely periodic structure supports surface modes, localized modes on the edge of a terminated surface are also supported when the layers comprising the structure are placed so that it there is just one overlap region per edge patch. This edge mode has been used to successfully guide the fields around both a triangular and a hexagonal shape. It has been experimentally shown that the structure is capable of supporting the edge mode in two different situations: When a hole is cut out of an infinite sheet and around a shaped metasurface embedded in a dielectric medium.

\section{ACKNOWLEDGEMENT}

We acknowledge financial support from the Engineering and Physical Sciences Research Council (EPSRC) of the United Kingdom, via the EPSRC Centre for Doctoral Training in Metamaterials (Grant No. EP/L015331/1). J.P.G. wishes to thank Flann Microwave Ltd (Bodmin, UK) for additional financial support of her studentship. All data created during this research are openly available from the University of Exeter's institutional repository at https://ore.exeter.ac.uk/repository/
[1] R. H. Ritchie, Phys. Rev. 106, 874 (1957).

[2] C. C. Cutler, in Proceedings of IEEE Antennas and Propagation Society International Symposium and URSI National Radio Science Meeting, Vol. 3 (1994) pp. 14561459.

[3] A. A. Oliner and A. Hessel, IRE Transactions on Antennas and Propagation 7, 201 (1959).

[4] J. B. Pendry, L. Martín-Moreno, and F. J. Garcia-Vidal, Science 305, 847 (2004).

[5] A. Hibbins, B. Evans, and J. Sambles, Science 308, 670 (2005).

[6] J. B. Pendry, D. Schurig, and D. R. Smith, Science 312, 1780 (2006).

[7] S. Maci, G. Minatti, M. Casaletti, and M. Bosiljevac, IEEE Antennas and Wireless Propagation Letters 10, 1499 (2011).

[8] J. A. Dockrey, M. J. Lockyear, S. J. Berry, S. A. R. Horsley, J. R. Sambles, and A. P. Hibbins, Physical Review B 87, 1 (2013).

[9] J. D. de Pineda, R. C. Mitchell-Thomas, A. P. Hibbins, and J. R. Sambles, Applied Physics Letters 111, 211603 (2017).

[10] G. Minatti, M. Faenzi, E. Martini, F. Caminita, P. De Vita, D. Gonzalez-Ovejero, M. Sabbadini, and S. Maci, IEEE Transactions on Antennas and Propagation 63, 1288 (2015).

[11] A. Hessel, A. A. Oliner, M. H. Chen, and R. C. Li, Proceedings of the IEEE 61, 183 (1973).

[12] O. Dahlberg, R. C. Mitchell-Thomas, and O. QuevedoTeruel, Scientific Reports 7, 10136 (2017).

[13] R. C. Mitchell-Thomas, J. R. Sambles, and A. P. Hibbins, 11th European Conference on Antennas and Propagation (EUCAP), 1396 (2017).

[14] O. Quevedo-teruel, M. Ebrahimpouri, and M. N. M. Kehn, IEEE Antennas and Wireless Propagation Letters 15, 484 (2016).

[15] J. D. de Pineda, R. C. Mitchell-Thomas, A. P. Hibbins, and J. R. Sambles, in 2017 IEEE International Symposium on Antennas and Propagation USNC/URSI National Radio Science Meeting (2017) pp. 831-832.

[16] M. Camacho, R. C. Mitchell-Thomas, A. P. Hibbins, J. R. Sambles, and O. Quevedo-Teruel, Opt. Lett. 42, 3375 (2017).

[17] C. Pfeiffer and A. Grbic, IEEE Transactions on Antennas and Propagation 58, 3055 (2010).

[18] M. Bosiljevac, M. Casaletti, F. Caminita, Z. Sipus, and S. Maci, IEEE Transactions on Antennas and Propaga- tion 60, 4065 (2012).

[19] T. Ma, A. B. Khanikaev, S. H. Mousavi, and G. Shvets, Phys. Rev. Lett. 114, 127401 (2015).

[20] A. Mekis, J. C. Chen, I. Kurland, S. Fan, P. R. Villeneuve, and J. D. Joannopoulos, Phys. Rev. Lett. 77, 3787 (1996).

[21] Z. Qiao, J. Jung, C. Lin, Y. Ren, A. H. MacDonald, and Q. Niu, Phys. Rev. Lett. 112, 206601 (2014).

[22] R. D. Meade, K. D. Brommer, A. M. Rappe, and J. D. Joannopoulos, Phys. Rev. B 44, 10961 (1991).

[23] A. B. Khanikaev, R. Fleury, S. H. Mousavi, and A. Alù, Nature Communications 6, 8260 (2015).

[24] A. B. Khanikaev and G. Shvets, Nature Photonics 11, 763 (2017).

[25] L. Lu, J. D. Joannopoulos, and M. Soljačić, Nature Photonics 8, 821 (2014).

[26] R. Collin, Field Theory of Guided Waves (IEEE Press, Piscataway, New Jersey, 1991).

[27] M. Camacho, R. R. Boix, F. Medina, A. P. Hibbins, and J. R. Sambles, Phys. Rev. B 95, 245425 (2017).

\section{Appendix A: Losses}

The propagation losses for the edge mode have been quantified over the range of frequencies where it is exclusively supported. We have chosen this range of frequencies because it is where the edge mode can be used for guiding purposes. For the same range of frequencies, the mode index has also been extracted from the experimental data. Between $19 \mathrm{GHz}$ and $27 \mathrm{GHz}$, the mode index varies between 1.11 and 1.43 while the decay constant of the mode normalized to the free space wave vector varies between 0.014 and 0.031 . This means that the wave has to travel for more than 50 wavelengths before its amplitude is reduced by a factor of e. The variation of the effective mode index and the normalized decay constant are shown in Fig. 8.

The loss due to radiation in the corner has also been quantified over the same range of frequencies. For frequencies between $19 \mathrm{GHz}$ and $22 \mathrm{GHz}$ the losses at the corner are smaller than $30 \%$, however as the frequency increases this percentage also increases. The amplitude losses at the corner are also shown in Fig 8. for the studied range of frequencies. 

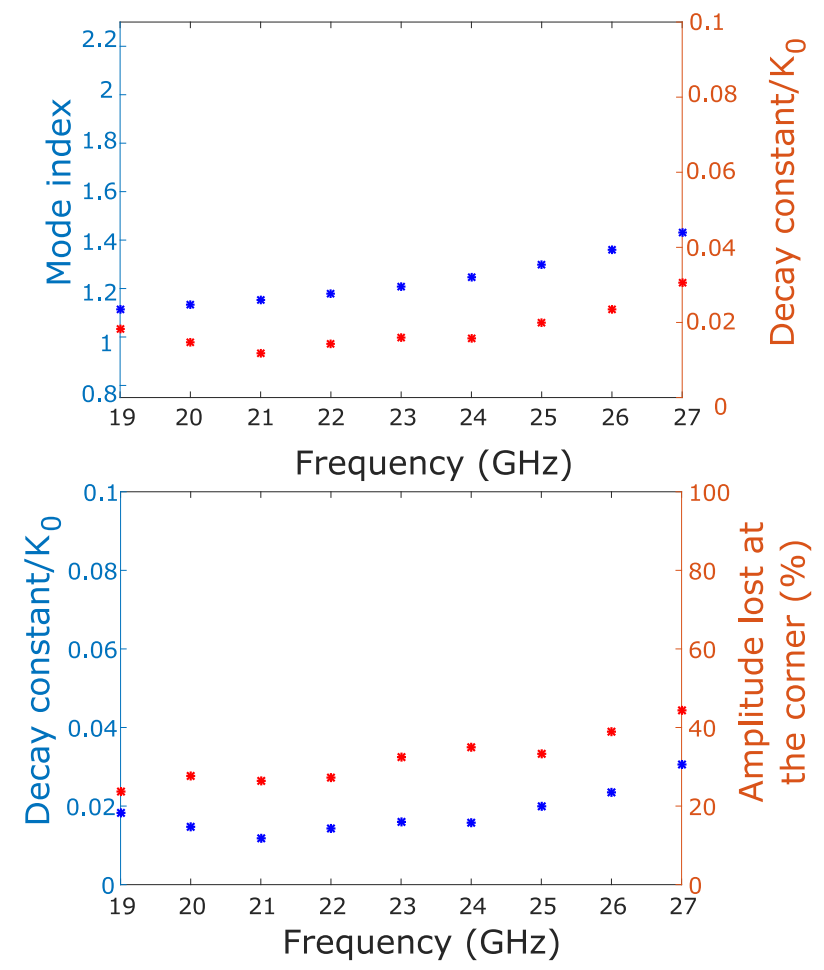

FIG. 8. Top: Variation of the mode index and the decay constant of the edge mode with frequency. For the range of frequencies where the edge mode is the only mode supported by the structure $(19 \mathrm{GHz}$ and $27 \mathrm{GHz})$, the mode index varies between 1.11 and 1.43 while the decay constant varies between 0.014 and 0.031. Bottom: Decay constant, normalized to the free space wave vector and amplitude lost at the corner for different frequencies in which the edge mode is supported. 